\title{
Introductions, réintroductions : des convergences, par-delà les différences
}

\author{
Isabelle Mauz \\ Sociologue, CEMAGREF, UR DTM, 2 rue de la Papeterie, BP 76, 38402 Saint-Martin-d'Hères cedex, France
}

Mots-clés :

(ré)introduction;

faune sauvage ;

sociologie;

Alpes françaises

\section{Keywords:}

re-introduction; wildlife;

sociology;

French Alps

\begin{abstract}
Résumé - Les lâchers d'animaux sauvages, qui se sont multipliés dans les Alpes au cours des dernières décennies, sont au premier abord très hétérogènes. Les protecteurs et les gestionnaires de la nature le soulignent, en distinguant notamment sans cesse introductions et réintroductions. Cette distinction mérite cependant d'être interrogée. Au-delà de leurs différences, ces opérations présentent en effet plusieurs points communs. Toutes procurent à leurs promoteurs le sentiment de vivre une expérience hors du commun. Toutes induisent une relation particulière à l'animal. Toutes manifestent l'existence de projets collectifs, partagés par certains mais rejetés par d'autres, et génèrent de ce fait de nouvelles proximités et de nouvelles tensions. Leurs convergences et l'influence qu'elles exercent sur les rapports des hommes entre eux plaident pour qu'elles ne soient pas menées comme si elles étaient indépendantes les unes des autres, ni comme s'il ne s'agissait que d'interventions purement techniques.
\end{abstract}

\begin{abstract}
Introductions, re-introductions: convergences, beyond differences. At first sight, operations consisting in releasing wild animals, which have become more and more common during the last decades in the French Alps, seem very heterogeneous. Nature protectors and managers of protected areas insist on their differences, and keep making a sharp distinction, in particular, between introductions, which they firmly condemn, and re-introductions, which they sometimes undertake. However, this distinction deserves to be questioned. Even if the methods, the results, the aims, the species and the persons involved are indeed different, these operations do share some common points, which deserve to be stated. All of them make their promoters feel that they are living an uncommon adventure. All generate a particular relation to the animal, which at the same time resembles that of hunters, farmers and nature observers, without being exactly the same as any of them. All show the existence of collective projects (on the animal, and on space, shared by some but rejected by others, who regard their own projects as threatened. These operations therefore produce new proximities and new tensions with persons that have not always been warned nor associated. Because of their similarities and of their influence on relations between social groups, they should not be carried out as if they were independent from one another, nor as if they were merely technical interventions.
\end{abstract}

Qui se rend aujourd'hui dans les Alpes françaises peut rencontrer, par exemple, des mouflons en Vanoise, des bouquetins dans le Queyras, des vautours fauves dans le Vercors, des castors et des gypaètes en HauteSavoie, des cerfs et des chevreuils un peu partout. Tous ces animaux ont fait l'objet de (ré)introductions au cours des dernières décennies. Davantage d'espèces et de régions ont été progressivement impliquées dans ces opérations et il n'est plus guère de massifs où au moins l'une d'elles n'ait été réalisée, envisagée ou ne soit projetée. Si la grande faune sauvage s'est fortement développée et sensiblement diversifiée dans les Alpes, les (ré)introductions n'y ont pas peu contribué.

Auteur correspondant : isabelle.mauz@grenoble.cemagref.fr
Il importe, dans ce contexte, de s'interroger sur ces pratiques et les changements qu'elles induisent. Deux idées simples me semblent ici essentielles. Premièrement, aucune intervention sur l'animal, même ponctuelle, ne se résume à sa dimension matérielle, ou technique. Pour (ré)introduire des animaux, il faut les capturer, ou les élever, les transporter, les relâcher, ce qui suppose de maîtriser des gestes, de forger des outils, et des mots, qui ne sont pas sans exercer des effets sur la relation de l'homme à l'animal dans son ensemble. Deuxièmement, les rapports des hommes entre eux ne sont pas indépendants de leurs liens aux non-humains en général, et aux animaux en particulier. Par conséquent, on ne peut modifier ceux-ci sans modifier ceux-là. Il ne suffit donc pas de chercher à savoir 
si les (ré)introductions génèrent une relation spécifique à l'animal. Il faut encore se demander si, et comment, elles affectent les rapports entre les différents groupes sociaux qui s'intéressent à la faune sauvage.

Des recherches menées dans les Alpes depuis plusieurs années sur les rapports aux animaux sauvages et sur l'histoire des espaces protégés m'ont amenée à rencontrer des naturalistes, des agents d'espaces protégés, des chasseurs et des éleveurs. J'ai réalisé, auprès d'eux, de nombreux entretiens, durant lesquels la question des (ré)introductions a été régulièrement évoquée. J'ai ainsi recueilli un ensemble de propos sur lesquels s'appuiera, pour l'essentiel, l'analyse qui va suivre.

Il faut d'emblée préciser à quels types de (ré)introductions nous nous intéresserons ici. Il en existe, en effet, de plusieurs sortes. Les (ré)introductions d'espèces sauvages sont volontaires lorsque des animaux sont délibérément lâchés. Elles sont accidentelles lorsque des animaux s'échappent d'enclos où ils étaient maintenus captifs ou sont transportés par des hommes à l'insu de ces derniers. Seules les premières retiendront notre attention. Ce n'est pas tout. Certains animaux sauvages, notamment des reptiles et des arachnides qui servent de «nouveaux animaux de compagnie », sont libérés dans l'intention de s'en débarrasser. D'autres, ceux qui nous occuperont dans cet article, sont lâchés dans un but bien différent : on espère qu'ils trouveront un environnement favorable et qu'ils constitueront, à terme, une population viable.

\section{Réintroductions et introductions : une proximité souvent déniée, mais réelle}

Il faut encore expliquer le choix de traiter ensemble les réintroductions et les introductions. Rappelons que les premières consistent à essayer «d'implanter une espèce dans une zone qu'elle occupait autrefois mais d'où elle a été éliminée ou d'où elle a disparu » (UICN, 1998), tandis que les secondes concernent des espèces qui n'y avaient jamais été présentes, ou dont la présence est du moins extrêmement ancienne, ou contestée. Or, les naturalistes, les protecteurs et les gestionnaires de la nature insistent sur la nécessité de bien distinguer les deux types d'opérations ${ }^{1}$. Seules les réintroductions trouvent grâce à leurs yeux. Jean-Louis Michelot (1991) titre la première partie de la conclusion de son rapport sur les réintroductions animales en Rhône-Alpes : «Pour en finir avec les introductions ». Après avoir exposé les nombreux inconvénients des introductions, il explique :

«En écologie, la définition la plus commune de la richesse d'un espace correspond à la diversité des espèces

\footnotetext{
${ }^{1}$ Cette insistance n'a pas échappé aux sociologues, qui l'ont à maintes reprises notée et commentée (Vourc'h et Pelosse, 1993 : 52 ; Micoud, 1993).
}

qui y vivent. L'introduction d'une espèce dans une niche écologique disponible entraînerait alors un enrichissement du milieu, tout à fait souhaitable. De nombreux chasseurs, pêcheurs et même naturalistes souscrivent à cette conception et approuvent par exemple les empoissonnements des lacs de montagne, les lâchers de marmottes dans le Massif Central, de mouflons dans les Alpes du Sud...

Nous ne sommes pas d'accord avec cette façon de penser. Nous pensons que les réintroductions doivent reconstituer le patrimoine que nous avons amputé et non construire une nature "riche" mais artificielle; en fait, réduire la richesse écologique à la seule diversité nous semble, sinon une erreur, du moins une conception qu'il est nécessaire de préciser. [...]

Sur le plan de l'éthique, nous pensons que la répartition des espèces doit témoigner de l'histoire climatique, géologique ou biologique d'une région. Même modifiée indirectement par des millénaires d'activités humaines, la nature doit rester à nos yeux un domaine non manipulé directement par les apprentis sorciers que nous sommes tentés d'être (op. cit. : 210). »

Cependant, la nécessité de distinguer introductions et réintroductions ne va pas de soi. Les sociétés de protection de la nature dérivent de sociétés d'acclimatation, qui ont été à l'initiative de l'introduction de toutes sortes d'espèces animales et végétales exotiques. Au début des années 1960, l'opportunité de lâcher des castors dans les hautes vallées savoyardes était âprement discutée au sein du comité scientifique du parc national de la Vanoise. Le projet fut finalement abandonné, mais que le débat ait eu lieu montre que l'exigence d'indigénat ne s'était pas encore totalement imposée. Outre qu'elle est relativement récente ${ }^{2}$, cette exigence est assez étroitement circonscrite à la sphère naturaliste. Bien des chasseurs ont ainsi participé à des introductions et à des réintroductions. En réalité, ils partagent avec les réintroducteurs stricts l'idée que toute espèce n'est pas bonne à lâcher, mais les critères d'éligibilité qu'ils retiennent sont autres : il faut, estiment-ils, que l'animal soit, et demeure, suffisamment farouche. Certaines espèces satisfont l'un et l'autre critères. Les cerfs et les chevreuils, qui sont chassés, sont relativement craintifs et, étant autochtones, ils ne dérogent pas à la règle naturaliste. $\mathrm{D}^{\prime}$ autres ne remplissent que l'un d'entre eux. Les bouquetins sont indigènes, mais de nombreux chasseurs les trouvent beaucoup trop tolérants à l'égard de l'homme et estiment qu'il faudrait "leur réapprendre à devenir sauvages ». Les mouflons sont dans une situation symétrique. Les chasseurs, qui les

\footnotetext{
2 En donnant des exemples de catastrophes écologiques causées par des nouveaux venus, l'ouvrage du naturaliste anglais Charles Elton (1900-1991) The ecology of invasions by animals and plants, paru en 1958, a largement contribué à diffuser l'idée selon laquelle les introductions comportent des risques imprévisibles et devraient être proscrites (Davis et al., 2001).
} 
ont introduits dans de nombreux massifs, ne les trouvent nullement déplacés dans les Alpes. Les naturalistes, eux, regardent leur présence comme une faute, dont ils espèrent qu'elle sera un jour sanctionnée par la nature. Aussi le goût prononcé que les loups ont montré pour les mouflons en arrivant dans le Mercantour ne les a-t-il guère inquiétés, bien au contraire. Les lâchers de poissons dans les lacs d'altitude sont également condamnés et accusés de détériorer la qualité de l'eau et de porter atteinte à la microfaune indigène : l'empoissonnement est synonyme d'empoisonnement ${ }^{3}$.

Ces divergences sur les qualités que doit présenter l'animal pour être lâché résultent de l'évolution des rapports au sauvage, qui a abouti à la coexistence de deux conceptions distinctes (Micoud, 1993). Les chasseurs et les éleveurs tendent à opposer les animaux sauvages aux animaux domestiques et à attendre des premiers qu'ils se montrent aussi craintifs et rétifs que les seconds sont censés être familiers et dociles. Pour les naturalistes, le caractère sauvage n'a rien à voir avec le comportement de l'animal, qui peut bien être aussi familier qu'il l'entend, pourvu qu'il n'ait pas été amené par l'homme et que sa présence soit réputée «naturelle». Ces divergences témoignent de rapports à la nature sensiblement différents. Les réintroducteurs veulent restaurer un milieu, en lui rendant une espèce qui vient théoriquement combler un manque, tandis que les introducteurs espèrent l'enrichir et l'améliorer, en y implantant une espèce qu'il n'avait a priori jamais hébergée. Les divergences entre introductions et réintroductions sont donc réelles. Il m'a cependant semblé qu'elles ne devaient pas masquer leurs proximités et qu'il ne faudrait pas oublier qu'il s'agit, dans tous les cas, de lâcher dans un milieu des animaux qui en étaient absents, et qui vont le coloniser, s'ils y parviennent.

\section{La grande hétérogénéité des (ré)introductions, de leurs promoteurs et des espèces impliquées}

L'étude des (ré)introductions se heurte très vite à une difficulté majeure : l'hétérogénéité des situations. Hétérogénéité, d'abord, de la réalité, des modalités pratiques et des résultats de ces opérations. Les unes sont tout à fait officielles et revendiquées. C'est le cas de la réintroduction du gypaète, coordonnée par Asters ${ }^{4}$ dans tout l'arc

\footnotetext{
${ }^{3}$ Les xénophytes ont trouvé leur champion en la personne du paysagiste Gilles Clément, qui a rappelé les raisons de leur succès et défendu leurs mérites. Mais son Éloge des vagabondes (Clément, 2002), à ma connaissance, n’a pas d'équivalent pour les animaux.

4 Asters (Agir pour la sauvegarde des territoires et des espèces remarquables ou sensibles) est le conservatoire d'espaces naturels de la Haute-Savoie.
}

alpin. D'autres ont été envisagées, plus ou moins sérieusement, avant d'être finalement abandonnées. Le projet «Ours Vercors » des années 1990 en est un bon exemple. D'autres encore sont simplement suspectées par certains, mais fermement démenties par les différentes structures impliquées dans les opérations de (ré)introduction. Je songe aux rumeurs de lâchers de grands prédateurs, en particulier de loups. Je tiendrai surtout compte, ici, des (ré)introductions avérées. Mais celles qui ont été projetées ou soupçonnées ne sont sûrement pas à négliger : les (ré)introductions auxquelles on a renoncé, comme celles dont on accuse les autres, disent quelque chose des (ré)introductions en général et de la façon dont elles sont perçues. Les techniques de (ré)introductions sont elles aussi variables. Après avoir vainement recouru à l'élevage, les promoteurs de la réintroduction du bouquetin se sont dirigés vers des captures en milieu naturel, suivies, aussi tôt que possible, par des lâchers. La réintroduction du gypaète a connu un cheminement inverse : des captures à l'étranger s'étant soldées par des échecs, les zoos détenteurs du rapace ont été sollicités afin de former des couples et d'obtenir des jeunes maintenus quelque temps en captivité avant d'être lâchés. Des (ré)introductions ont été de véritables fiascos, comme celle de ces bouquetins, capturés en Haute-Maurienne en 1981 et réintroduits à Pralognan, qui s'empressèrent de retourner chez eux. Beaucoup d'entre elles ont cependant réussi et les objectifs visés par leurs promoteurs ont souvent été atteints, voire dépassés ${ }^{5}$. Hétérogénéité, ensuite, des espèces (ré)introduites. Elles diffèrent par leur statut juridique. Les unes sont protégées, comme le bouquetin, le gypaète, et le castor, tandis que d'autres sont chassées, comme le cerf, le chevreuil et le mouflon. Elles diffèrent par leur statut biologique. À côté d'espèces encore très peu représentées, comme le gypaète, $\mathrm{d}$ 'autres sont devenues relativement communes, dans certaines régions au moins et dans des espaces protégés surtout. Plus de deux mille bouquetins ont ainsi été recensés dans la zone centrale du parc national de la Vanoise en 2003. Les contrastes sont également flagrants si l'on considère le comportement des animaux et leur régime alimentaire. Parmi les espèces (ré)introduites, on trouve à la fois un rongeur comme le castor, des herbivores comme le cerf, le chevreuil, le bouquetin, le mouflon, un prédateur comme le chat sauvage, ou encore un oiseau essentiellement ostéophage, comme le gypaète. Aussi les (ré)introductions ont-elles des conséquences très différentes sur le fonctionnement des écosystèmes. Si l'impact d'un lâcher de gypaètes est a priori limité, la plupart des animaux impliqués sont des proies, des prédateurs, ou

\footnotetext{
${ }^{5}$ Selon l'enquête menée par Griffith et al. (1989), principalement en Amérique du Nord, en Australie et en Nouvelle Zélande, moins de la moitié (44\%) des translocations conduites avec des oiseaux et des mammifères sensibles ou menacés ont permis l'établissement d'une population viable.
} 
des concurrents pour des espèces déjà présentes. Corrélativement, il est fréquent qu'ils influencent les activités humaines, ce qui retentit sur la façon dont ils sont considérés. Ils sont susceptibles de causer des dégâts de nature et d'ampleur très variables. Ils peuvent aussi induire des effets positifs, en régulant des populations d'autres espèces posant elles-mêmes des problèmes, argument qui a parfois été avancé en faveur de la réintroduction de grands prédateurs, ou en favorisant l'apparition ou le développement d'activités cynégétiques ou touristiques. Hétérogénéité, enfin, des (ré)introducteurs et de leurs motivations. Étant bien entendu qu'il s'agit d'un axe de polarité comprenant toute une série d'intermédiaires, on peut distinguer deux grands objectifs. Celui des naturalistes, des protecteurs et des gestionnaires de la nature est avant tout de reconstituer «la » nature. Celui des chasseurs, et des pêcheurs, est d'obtenir, un jour, des populations dans lesquelles effectuer des prélèvements.

\section{D'une opération à l'autre, des convergences}

Des points communs à toutes ces entreprises peuvent cependant être dégagés qui, peut-être, l'emportent sur leurs différences. Trois d'entre eux semblent particulièrement importants.

\section{Un commencement}

Les (ré)introductions sont, dans l'ensemble, des opérations extrêmement délicates. Une multitude d'obstacles et de problèmes doivent être surmontés. Il faut vaincre des réticences, obtenir des autorisations et des appuis, se procurer et transporter des animaux dont les besoins et les préférences sont, par définition, méconnus. Sans doute est-ce moins vrai lorsqu'une espèce est (ré)introduite depuis longtemps, le recul que confèrent les expériences et les déboires antérieurs permettant de savoir comment procéder et ce qu'il faut éviter. Il n'empêche qu'une (ré)introduction reste toujours une aventure.

Il faut entendre le terme dans son acception usuelle. Les récits de (ré)introductions sont généralement pleins d'épisodes imprévus, heureux ou malheureux, parfois franchement rocambolesques. Au début du $20^{\mathrm{e}}$ siècle, des braconniers ont été engagés par l'administration helvétique pour aller subtiliser des bouquetins dans le Grand Paradis, seul massif où l'espèce subsistait, et les ramener en Suisse. Des gypaètes ont été capturés sur des dépôts d'ordures afghans et transportés jusqu'en Haute-Savoie, via l'aéroport de Beyrouth. La réintroduction du gypaète s'est avérée particulièrement difficile et riche en péripéties. En voici un exemple, relaté par un de ses artisans :

«Le plus vieux des trois couples que j'avais, a été long à procréer. La femelle avait dix-huit ans quand elle a pondu un premier œuf et, ça arrive, il n'était pas fécondé. La deuxième ponte, il y a eu deux œufs et le deuxième œuf a éclaté dans l'oviducte. Il a créé une septicémie et j'ai perdu ma femelle. Le premier œuf restait là et je n'avais plus que le mâle. Je ne pouvais pas mettre une autre femelle parce que je savais que ça allait mal se passer. J'ai téléphoné à Hans Frey ${ }^{6}$ qui m'a dit : "vous m'envoyez tout de suite l'œuf par avion". J'ai emmené l'œuf à Genève et c'est l'Austria Airlines qui a accepté de le prendre dans la cabine de pilotage. Je suis arrivé là-bas, avec mon paquet bien enveloppé - l'œuf supportait les variations de température mais il fallait le protéger des chocs, bien entendu. Les douaniers ont voulu ouvrir le paquet et j'ai retardél'avion de dix minutes. Mon œuf est bien parti, il est bien arrivé, il a vécu jusqu'à cinq jours de l'éclosion et, manque de chance, le poussin est mort dans l'œuf. Voyez un peu le genre d'histoires incroyables qu'on a vécues. On a essuyé les plâtres. De toute façon, on était, je ne dirais pas des apprentis sorciers, mais presque. Personne ne connaissait cet oiseau à fond. »

Sans doute l'odyssée tragique de cet œuf constitue-telle un cas extrême. Mais elle montre bien l'esprit pionnier des (ré)introducteurs : ils ont dû se «débrouiller", trouver des solutions au pied levé, faire preuve de détermination et d'audace.

Cependant, ce ne sont pas tant les difficultés qu'ils ont rencontrées ni les échecs qu'ils ont essuyés qui dominent leurs récits, que l'enthousiasme et l'exaltation qui, presque toujours, marquent une naissance. Toute (ré)introduction est un commencement, une aventure, cette fois au sens étymologique de ce qui est à venir. De ce point de vue, peu importe que l'animal n'ait plus ou pas été là : à compter du moment inaugural du lâcher, quelque chose qui n'existait pas se met à exister. Un ancien réintroducteur raconte :

«Dans les années 1970, on a lâché sept bouquetins et ces bouquetins je les ai longtemps suivis. J'ai vu la harde grossir tout doucement [...]. Et puis je suis parti, et voyezvous, il y a à peu près trois ou quatre mois ${ }^{7}$, je me suis demandé : "tiens, au fait, ces bouquetins, qu'est-ce qu'ils sont devenus ?" Alorsj'ai téléphoné au bureau des guides et j'ai demandé à parler à l'un d'eux, n'importe lequel, parce que les vieux que je connaissais, ils sont comme moi, ils sont à la retraite. On m'a passé un guide qui a été extrêmement aimable : - "Vous voyez toujours les bouquetins ?" - "Oui" - "Vous les voyez où ?" Ils étaient toujours dans le même secteur. - "Ils sont combien?" - "Oh, des fois, on en voit au moins une bonne vingtaine." Alors j'ai dit: "Ben je vous remercie, ça me fait plaisir". Il m’a demandé pourquoi je lui avais posé ces questions

\footnotetext{
${ }^{6}$ Directeur de l'institut de médecine vétérinaire de l'université de Vienne en Autriche et responsable du programme d'élevage en captivité et de réintroduction du gypaète barbu.

7 L'entretien a eu lieu en mars 2003, soit 30 ans, environ, après la réintroduction.
} 
alors je lui ai expliqué et il m'a dit : "Ah, c'est une belle chose que vous avez faite".»

Les bouquetins sont très lents à coloniser de nouveaux territoires et ils ne seraient probablement toujours pas dans le massif en question s'ils n'y avaient été amenés : leur présence s'explique entièrement par la réintroduction. De manière générale, les (ré)introducteurs sont à l'origine d'une histoire radicalement nouvelle dont ils peuvent revendiquer la paternité. Ce n'est, après tout, pas si fréquent. Il n'est pas surprenant, dans ces conditions, que leurs récits et leurs projets prennent si souvent la forme du mythe, comme l'a remarqué Sophie Bobbé à propos de l'ours (2002 : 164-169). Le mythe désigne une rupture temporelle. La distinction d'un avant et d'un après, nettement séparés par un événement clairement identifié, auquel se rattache tout ce qui en découle, est même l'une de ses caractéristiques essentielles (Cassirer, 1972 : 132). Il en va de même avec les (ré)introductions.

\section{Une relation particulière entre l'homme et l'animal}

Les animaux (ré)introduits ont pour autre caractère commun d'avoir tous été étroitement en contact avec des hommes. Ce contact est plus ou moins prolongé selon les espèces. Bref en cas de capture dans le milieu naturel, il peut durer plusieurs semaines, voire plusieurs mois, si l'opération comporte une phase d'élevage, éventuellement suivie d'une phase d'acclimatation progressive au milieu du lâcher. Quelle que soit la technique, l'animal est manipulé. En Vanoise, les membres de la première génération d'agents de terrain les plus impliqués dans les captures de bouquetins étaient tous d'anciens chasseurs de chamois. Sans doute retrouvaient-ils, dans le tir au fusil téléanesthésique et dans le transport de l'animal endormi, des expériences voisines de celles qu'ils avaient éprouvées, et aimées, avant d'entrer au parc, en tant que chasseurs. Les réintroductions offrent aux naturalistes, aux protecteurs et aux gestionnaires de la nature l'opportunité unique de toucher les animaux, sans mauvaise conscience de surcroît, puisqu'ils ont la certitude d'agir «pour la bonne cause». Pour une fois, leur rapport à l'animal n'est pas un rapport à distance, mais un véritable corps à corps. Ce n'est d'ailleurs pas un hasard si les espèces (ré)introduites sont, pour l'essentiel, de grands mammifères et de grands oiseaux, alors qu'ils ne sont ni les seuls à avoir disparu, ni les plus faciles à (ré)installer ${ }^{8}$. Simplement, leur observation et leur contact procurent

\footnotetext{
${ }^{8}$ Une étude récente (Seddon et al., 2005) met en évidence un important «biais taxonomique » dans les projets de réintroduction : la comparaison de la distribution des projets de réintroduction et de la proportion des espèces connues montre que les mammifères et les oiseaux sont fortement surreprésentés. Parmi les mammifères, on observe une surreprésentation des artiodactyles et des carnivores, groupes riches en espèces emblématiques.
}

généralement davantage de plaisir que ceux d'autres espèces. Des insectes et des reptiles ont certes été réintroduits mais pas n'importe lesquels : un beau papillon, l'apollon, réintroduit non dans les Alpes mais dans les Vosges, une tortue, la tortue cistude, réintroduite au sud du lac du Bourget (Savoie). Si le contact physique s'interrompt avec le lâcher, une relation privilégiée est maintenue avec les animaux (ré)introduits, puisqu'ils sont fréquemment équipés de dispositifs permettant de les localiser, de les identifier, et de retarder le moment où le lien avec les (ré)introducteurs est rompu (Larrère, 1994). Le «baptême » des animaux, largement répandu et systématique pour certaines espèces comme le gypaète, illustre et renforce l'attachement des (ré)introducteurs pour les animaux qu'ils ont eus entre les mains. Le (ré)introducteur noue ainsi une relation particulière à l'animal, qui n'est ni celle de l'éleveur, ni celle du chasseur, ni celle du protecteur, mais qui, par certains aspects, ressemble à chacune d'elles : il nomme l'animal, comme l'éleveur, le capture, comme le chasseur, veille sur son existence, comme le protecteur.

Le plaisir du (ré)introducteur n'est pas nécessairement partagé par l'animal et l'opération n'est pas, pour lui, dénuée de dangers. Le taux de mortalité des bouquetins au cours des réintroductions a longtemps été non négligeable, des individus succombant durant la capture ou le transport. De nombreuses substances anesthésiantes et divers modes de transport ont été testés et des progrès significatifs ont été accomplis ${ }^{9}$. Les bouquetins sont aujourd'hui téléanesthésiés, transportés jusqu'à un véhicule, réveillés, un ruminant ne pouvant rester endormi trop longtemps, avant d'être acheminés jusqu'au site de lâcher, les yeux masqués d'un tissu noir. La mort de l'animal, toujours très mal vécue par les (ré)introducteurs, n'est pas le seul risque encouru. Des animaux (ré)introduits ont montré des comportements inattendus et fâcheux. Dans la première moitié du $20^{\mathrm{e}}$ siècle, le lâcher de bouquetins élevés en captivité a été un échec total : "c'était des animaux complètement imprégnés par l'homme, qui revenaient dans les villages, demandaient du pain, avaient un peu d'agressivité et pouvaient encorner les gens : ça n'a pas marché du tout ${ }^{10}$. » Deux des lynx réintroduits dans les Vosges dans les années 1980 n'ont pu être laissés en liberté, en raison de leur familiarité excessive avec les humains. Ces cas dits d'«imprégnation» ont accrédité l'idée que les animaux sauvages ne sortent pas indemnes du contact avec l'homme, que celui-ci les transforme, et jamais dans le bon sens : l'animal (ré)introduit est perçu comme

\footnotetext{
${ }^{9}$ Le taux de mortalité des bouquetins lors des captures, en Vanoise, est passé de 10,3 à 4,6 \% lorsque des projecteurs à air comprimé et des fléchettes en plastique ont remplacé les projecteurs à poudre et les fléchettes en aluminium (Gauthier, 2001).

10 Dominique Gauthier, com. pers.
} 
possiblement sinon probablement déviant. La majorité des éleveurs ovins sont ainsi persuadés que les loups ont été réintroduits dans les Alpes, du moins certains loups : ceux qui, ne craignant pas de s'approcher des troupeaux et des habitations, posent problème.

\section{L'animal (ré)introduit manifeste l'existence d'un projet collectif}

Troisième caractère commun aux animaux (ré)introduits : ils ne sont pas venus spontanément. Ils ne sont pas là non plus par hasard ni par accident mais parce que des hommes ont voulu et organisé leur installation. Ils manifestent l'existence d'un collectif doté d'un projet.

Une personne seule peut en effet difficilement se charger à la fois de la capture, du transport et du lâcher des animaux. Des collaborations et des «complicités » sont nécessaires. Le président des lieutenants de louveterie de Haute-Savoie est intervenu auprès des services de douane afin que les véhicules transportant les bouquetins ne soient pas arrêtés à la frontière franco-suisse. En Haute-Savoie toujours, des chasseurs se sont fortement impliqués dans la réintroduction du castor, du gypaète et du bouquetin, aux côtés de membres de l'administration, de gestionnaires d'espaces protégés et de naturalistes. Plusieurs opérations ont ainsi permis des rapprochements entre des individus appartenant à des groupes réputés s'opposer. Des techniques comme la pose de lacets, l'installation de trappes et de cages, et bien sûr le tir ont également circulé des uns aux autres.

Les (ré)introducteurs sont convaincus que leurs entreprises sont populaires parce qu'elles tranchent avec leurs pratiques ordinaires. Les protecteurs sont régulièrement accusés d'être des chantres de l'inertie et de l'immobilisme. En réintroduisant des animaux, ils prouvent qu'ils peuvent avoir une démarche active et prendre les choses en main. Les chasseurs, eux, n'apparaissent plus seulement comme des tueurs, mais comme des gens capables d'exercer une action positive, y compris lorsque le bénéfice pour la pratique cynégétique semble lointain ou très incertain.

Si le grand public assiste en effet volontiers à des opérations souvent spectaculaires et présentées comme des "fêtes de la nature ", l'étroite association des (ré)introductions à des projets soulève pourtant des interrogations et peut créer des tensions, voire des conflits. Il se trouve d'abord des gens pour remarquer que, l'erreur étant humaine, les animaux peuvent être lâchés dans un site, à un moment ou avec des partenaires qui ne leur conviennent pas vraiment. Les gypaètes, à nouveau, se sont montrés particulièrement difficiles. Comme ce sont des oiseaux sans dimorphisme sexuel apparent, former des couples de géniteurs n’a pas été une sinécure. Lorsqu'on y est enfin parvenu, les oiseaux appariés ne se sont pas toujours entendus et il a parfois fallu les séparer. Certes, les problèmes pratiques s'aplanissent généralement à mesure que les (ré)introducteurs apprennent à mieux connaître l'animal. Les conditions dans lesquelles sont entreprises les (ré)introductions sont en outre définies beaucoup plus précisément que par le passé. Reste en revanche la question de principe, qui tracasse certains naturalistes et protecteurs : peut-on intervenir dans les processus naturels? Un agent d'un espace protégé explique sa décision de cesser de s'impliquer dans la réintroduction du bouquetin : «Maintenant, avec du recul, je me dis : on a joué les apprentis sorciers alors que le phénomène allait se passer naturellement. Au niveau de l'éthique on aurait dû se passer de cette manip, alors que la colonisation du bouquetin s'est faite en quelques années. »

La question des objectifs et des effets des (ré)introductions est plus épineuse encore. Car ce ne sont pas seulement les animaux qui sont (ré)introduits mais, simultanément, les attributs qui leur sont associés. Or, ces attributs se communiquent aux lieux où ils se trouvent, ce qui est, à nouveau, un trait de la pensée mythique (Cassirer, 1972 : 119). Le projet sur l'animal est indissolublement un projet sur l'espace. Nous avons vu que les (ré)introducteurs ne sont pas tout à fait d'accord sur les qualités de l'animal : un lâcher de bouquetins naturalise la montagne aux yeux des naturalistes, des gestionnaires et des protecteurs de la nature, mais pas des chasseurs, tandis que ces derniers attendent plutôt des cerfs, des chevreuils ou des mouflons qu'ils l'ensauvagent. Les éleveurs, eux, voient dans ces divergences des subtilités qui masquent l'essentiel : les Alpes s'ensauvagent à un rythme accéléré et la présence d'espèces (ré)introduites est la preuve patente de l'existence d'une volonté partagée par beaucoup. Ils ne considèrent pas les (ré)introductions comme dissociées les unes des autres mais comme les éléments d'un projet global et cohérent. La réflexion du président de la fédération ovine des Alpes maritimes à propos du gypaète, dans le cadre de l'enquête parlementaire sur l'origine des loups en France, est à cet égard tout à fait éloquente : "Quand, en 1990, le gypaète - grand rapace de trois mètres d'envergure qui ne mange que des os - a été introduit, nous nous sommes demandés d'où viendraient les os. Il nous a été répondu qu'il y en aurait. En fait, les personnes qui nous ont répondu savaient bien ce qui se préparait! Et leurs propos sont restés marqués dans nos mémoires ${ }^{11}$. »

L'histoire des réintroductions montre plutôt une série d'initiatives disjointes (Bigan et Simon, 2000). Les

\footnotetext{
11 Rapport de la commission d'enquête sur les conditions de la présence du loup en France et l'exercice du pastoralisme dans les zones de montagne, disponible sur http://www.assembleenationale.fr/12/rap-enq/r0825-t1-2.asp
} 
(ré)introductions se sont produites au gré des opportunités et des passions des uns et des autres. Si certains ont été à l'initiative de la (ré)introduction de plusieurs espèces, notamment Gilbert Amigues en Haute-Savoie, des noms sont souvent associés à la réintroduction d'une espèce particulière, ceux de Bernard Richard et de Maurice Blanchet pour le castor, des frères Terrasse et de Paul Géroudet pour le gypaète, ou celui de Dominique Gauthier pour le bouquetin. Il ne semble pas qu'il y ait eu de vision d'ensemble ni que les (ré)introductions aient été orchestrées. Mais elles ont bien eu, de fait, des effets convergents : la réintroduction de grands prédateurs a pu être envisagée parce que les grands ongulés sont devenus plus nombreux; les gypaètes barbus bénéficient incontestablement de la présence de nombreux ongulés et de grands prédateurs.

Mais revenons aux acteurs du pastoralisme. Ils ne seraient pas tellement convaincus de l'existence d'un projet d'ensauvagement des Alpes et ils ne lui seraient pas tellement hostiles s'il n'existait une autre entreprise, vieille de plusieurs siècles celle-là, de domestication de la montagne. Jusqu'à très récemment, les Alpes, leurs habitants et les troupeaux domestiques formaient un tout indissociable. Dans L'Homme et la montagne (Blache, 1934), le géographe Jules Blache définissait les montagnes en général, et les Alpes en particulier, comme le domaine des migrations pastorales. Parallèlement, les sociétés alpines ont été qualifiées de « sociétés de la vache » (Poche, 1999) ou, dans une moindre mesure, du mouton ou de la chèvre, en tout cas de l'ongulé domestique. Cette domestication s'est opérée en introduisant des espèces domestiques, en éradiquant les espèces sauvages les moins facilement conciliables avec l'activité agropastorale et en maintenant les autres en deçà d'un seuil extrêmement faible. Les sociétés alpines ont condamné les animaux sauvages à demeurer rares et farouches, notamment par un exercice de la chasse, qui ne s'encombrait pas de gestion des populations animales sauvages (Dalla Bernadina, 1989). Les (ré)introducteurs d'espèces sauvages font très précisément le contraire de ce qu'ont fait les montagnards qui ont domestiqué la montagne. Aussi leur action est-elle perçue comme une « dé- » ou une « antidomestication ».

Le fossé est tel, aujourd'hui, entre les (ré)introducteurs et les tenants d'une montagne domestiquée que le clivage entre les réintroducteurs et les introducteurs, les faiseurs de nature et les faiseurs de sauvage, semble, par contraste, plutôt secondaire. Les conflits entre chasseurs et éleveurs sont d'ailleurs aussi, sinon plus importants que ceux qui opposent les éleveurs aux protecteurs de la nature. Il y a quelques décennies encore, les montagnards étaient tous éleveurs et quelques-uns, parmi eux, chassaient. Aujourd'hui, les éleveurs sont très minoritaires et il est rare d'en trouver plus d'un ou deux parmi les cinquante à cent chasseurs que comptent la plupart des communes. Ils ne partagent plus les mêmes intérêts ni les mêmes préoccupations et les (ré)introductions de grands ongulés par les chasseurs ont accéléré et accentué leurs divergences, nettement perceptibles dans les propos de nombreux éleveurs. L'un d'eux s'exclame : "Maintenant, les chasseurs, eux, veulent qu'il y ait le maximum de cerfs, de biches, de sangliers, de toutes les bêtes. Ils n'ont pas de propriété, ils n'ont rien; c'est pas un problème, pour eux. Bon, ils payent un peu les dégâts. Mais quand $t^{\prime}$ as un pré qui est labouré complètement, ou qu'il n'y a plus d'herbe, que les cerfs ont tout mangé, c'est autre chose. » Les (ré)introductions ne sont évidemment pas le seul facteur à l'origine de l'augmentation de la grande faune sauvage : la protection de certaines espèces, la création d'espaces protégés, l'adoption des plans de chasse, l'abandon des terrains les moins favorables à l'agriculture et l'avancée des friches et de la forêt, y sont également pour beaucoup. Mais, de tous les processus qui ont contribué au développement de la grande faune sauvage dans les Alpes, il n'y en a aucun où la responsabilité des hommes soit plus directe ni plus flagrante que dans les (ré)introductions.

\section{Conclusion}

Que les (ré)introductions soient plus proches qu'il n'y paraît à première vue et qu'elles contribuent à reconfigurer les relations entre les acteurs impliqués dans la gestion des espaces montagnards n'est pas dénué de conséquences pratiques et politiques. Les (ré)introductions sont aujourd'hui bien mieux préparées que par le passé. Les scientifiques spécialistes du bouquetin ont par exemple élaboré une «charte » qui prescrit le déroulement des réintroductions de l'ongulé. Les facteurs de réussite des translocations d'oiseaux et de mammifères ont fait l'objet d'enquêtes successives (Griffith et al., 1989; Wolf et al., 1996) et le Groupe de spécialistes de la réintroduction de la Commission de la sauvegarde des espèces de 1'UICN a publié des lignes directrices, où sont formulées toute une série de recommandations (UICN, 1998). Aussi les opérations sont-elles à présent précédées d'une enquête qui examine si les conditions, non seulement écologiques mais aussi sociales, sont favorables à leur réalisation et elles sont parfois suivies d'une évaluation. La plupart du temps, cependant, les études menées reposent sur une vision assez étroite des personnes impliquées, qui mériterait d'être élargie : plus de personnes qu'on ne le croit sont intéressées par ces opérations. Par ailleurs, elles ne tiennent pas toujours compte de l'ensemble des espèces ayant déjà été (ré)introduites ou qui le sont par d'autres. Les (ré)introducteurs sont très souvent des (ré)introducteurs de bouquetins, ou de gypaètes, ou de mouflons, ou de castors, etc. : ils tendent à concentrer leurs efforts et leur attention sur l'espèce qui les passionne. Mais les (ré)introductions ne sont pas dissociables 
les unes des autres. Leurs effets écologiques et sociaux s'ajoutent et, pour les non-(ré)introducteurs, les animaux « qui ont été mis » forment un tout. Qu'une espèce soit la première ou la cinquième à être (ré)introduite n'est donc pas neutre; que ces opérations se soient étalées sur dix ans ou sur un demi-siècle non plus.

\section{Références}

Bigan, M., Simon, G., 2000. Histoire des réintroductions en France, Le Courrier de la nature, 182, 10-13 (Spécial Réintroductions).

Blache, J., 1934. L'Homme et la montagne, Paris, Gallimard, coll. Géographie humaine.

Bobbé, S., 2002. L'Ours et le loup. Essai d'anthropologie symbolique, Paris, MSH, Inra.

Cassirer, E., 1972. La Philosophie des formes symboliques. 2. La pensée mythique, Paris, Éd. de Minuit.

Clément, G., 2002. Éloge des vagabondes, Paris, Nil Éditions.

Dalla Bernadina, S., 1989. L'invention du chasseur écologiste : un exemple italien, Terrain, 13, 130-139.

Davis, M.A., Thompson, K., Grime, J.P., 2001. Charles S. Elton and the dissociation of invasion ecology from the rest of ecology, Diversity and Distributions, 7, 97-102.

Elton, C., 1958. The ecology of invasions by animals and plants. London, Methuen.
Gauthier, D., 2001. Bilan de 20 ans de capture par téléanesthésie au parc national de la Vanoise, Rencontres du Groupe d'études sur l'écopathologie de la faune sauvage de montagne, Vébron, 17-20 mai 2001.

Griffith, B., Scott, J.-M., Carpenter, J.W., Reed, C., 1989. Translocation as a species conservation tool: status and strategy, Science, 245, 477-480.

Larrère, R., 1994. Sauvagement artificiel, Le courrier de l'environnement de l'Inra, 21, 35-37.

Michelot, J.-L., 1991. Les réintroductions animales en Rhône-Alpes, FRAPNA / Région Rhône-Alpes.

Micoud, A., 1993. Vers un nouvel animal sauvage : le sauvage «naturalisé vivant »? Natures Sciences Sociétés, 1, 3, 202-210.

Poche, B., 1999. Le Monde bessanais. Société et représentation, Paris, CNRS éditions.

Seddon, P. J., Soorae, P. S., Launay, F., 2005. Taxonomic bias in reintroduction projects, Animal Conservation, 8, 51-58.

UICN, (1998). Lignes directrices de l'UICN relatives aux réintroductions. Préparées par le Groupe de spécialistes de la réintroduction de la Commission de la sauvegarde des espèces de l'UICN, Gland, Suisse et Cambridge, Royaume-Uni, UICN (Consultable sur www.iucn.org).

Vourc'h, A., Pelosse, V., 1993. Du bestiaire au paysage. (Ré)introduire des espèces oubliées, Études Rurales, 129-130, 51-58.

Wolf, C.M., Griffith, B., Reed, C., Temple, S.A., 1996. Avian and mammalian translocations: update and reanalysis of 1987 survey data, Conservation biology, 10, 1142-1154. 